\title{
Evaluation of the Volumizing Performance of a New Volumizer Filler in Volunteers with Age-Related Midfacial Volume Defects
}

This article was published in the following Dove Press journal: Clinical, Cosmetic and Investigational Dermatology

\author{
Adele Sparavigna (D) \\ Annalisa La Gatta ${ }^{2}$ \\ Gilberto Bellia (1D ${ }^{3}$ \\ Laura La Penna (D) \\ Andrea Maria Giori (iD ${ }^{4}$ \\ Gabriele Vecchi ${ }^{4}$ \\ Beatrice Tenconi (D) ${ }^{1}$ \\ Chiara Schiraldi $\mathbb{D}^{2}$ \\ 'DERMING Srl., Milan, Italy; \\ ${ }^{2}$ Department of Experimental Medicine, \\ Section of Biotechnology, Medical \\ Histology and Molecular Biology, School \\ of Medicine, University of Campania "L. \\ Vanvitelli”, Naples, Italy; ${ }^{3}$ Medical Affairs, \\ IBSA Farmaceutici Italia, Lodi, Italy; \\ ${ }^{4}$ Research and Development, IBSA \\ Farmaceutici Italia, Lodi, Italy
}

Correspondence: Adele Sparavigna DERMING Srl, Clinical Research and Bioengineering Institute, Via Valassina, 24, Milano, Italy

$\mathrm{Tel}+39223183475$

Email adele.sparavigna@derming.com
Purpose: The primary aim of this study was to evaluate the performance of the study product, in terms of volumizing activity as well as the duration of the effect, in women with age-related midfacial volume defects. In addition, the study allowed the evaluation of the tolerability of the product by both volunteers and investigators.

Patients and Methods: Twenty-two female volunteers, aged 42-60 years, participated in this study, which was performed under dermatological control in a single center. After an initial visit at baseline to verify adherence to the protocol criteria, volunteers received an injection of Aliaxin ${ }^{\circledR}$ SV (IBSA Farmaceutici Italia Srl), followed 3-4 weeks later by a second touch-up treatment to treat eventual asymmetries. Four subsequent visits, the last performed 9 months from the first injection, were performed to evaluate clinically and instrumentally the efficacy of the treatment.

Results: Clinical and statistically significant improvement in cheek volume was recorded after the first postinjection visit, and the effect was maintained until the end of the study period. A clinically measurable amelioration of wrinkle severity was also observed. By 3D picture recording and subsequent quantitative analysis, it was possible to determine the efficacy in terms of increased facial volume, which was already appreciable at the first visit, was further increased at the second and third visits and was maintained at the fourth and last visits. The injections were very well tolerated by the volunteers, as determined by their self-evaluation questionnaires.

Conclusion: The results of the study confirm the esthetic performance of the study product on age-related midfacial volume defects. The very strong high-volumizing activity of the study product was not only properly determined by the investigators but also confirmed by self-evaluation by the volunteers. These effects were obtained with no appreciable undesired effects.

Keywords: midfacial aging, crosslinked hyaluronic acid, clinical assessment, instrumental assessment

\section{Introduction}

Midfacial aging is a common, complex and multifactorial process that involves both intrinsic factors and extrinsic factors that, over time, act on several structural components, soft tissues and the skin. ${ }^{1-3}$ In recent decades, convincing evidence has been obtained that improved the understanding of the pathogenesis of the aging of the face and modified the approaches and treatments to revert this phenomenon. ${ }^{4-6}$ In particular, these age-related changes happen at different tissue layers, including the bone, fat dermis and epidermis, and the major effect is volume 
loss in the midface, which, in turn, leads to skin sagging and a deepening of the nasolabial folds. ${ }^{6-8}$ From these considerations, it is clear that the best possible option is to counteract these changes through a three-dimensional approach, and volumizer fillers represent an ideal minimally invasive treatment for midfacial aging. Among these fillers, crosslinked hyaluronic acid (HA)-based fillers are widely used as injectable materials able to increase midfacial volume and correct facial defects at the soft-tissue level. $^{9-13}$ Importantly, HA is found in all human connective tissue, where it lays in the extracellular space to act both as a structure stabilizer and as a cell protector. In the dermis, HA helps in maintaining skin turgor and elasticity due to its ability to bind water. ${ }^{9,14}$ Several HA-containing preparations differ in molecular mass, viscosity and concentration and can be either natural or crosslinked. For esthetic purposes, any preparation, including those containing HA, must be biocompatible, must have good tolerability and should produce a long-lasting effect.

Aliaxin $^{\circledR}$ SV (IBSA Farmaceutici Italia Srl) is a nonpyrogenic sterile gel composed of crosslinked HA of nonanimal origin produced by bacterial fermentation, and it is used as a filler to correct facial cutaneous sagging and increase facial volume. In this study, the product was tested for its performance and duration of effect as a volume augmenter in volunteer women aged between 40 and 65 years with age-related midfacial volume defects.

\section{Subjects and Methods}

Rheological parameters for the study product were measured with a physical oscillatory rheometer (MCR301, Anton Paar, Germany). Frequency sweep tests were carried out at $37^{\circ} \mathrm{C}$ with a PP geometry, $25 \mathrm{~mm}$ plate diameter, $1 \mathrm{~mm}$ gap, at $0.1 \%$ strain (within the Linear Viscoelastic Range) and frequency in the range of 0.159-$10 \mathrm{~Hz}$. Measurements were performed in triplicate. The results for the storage modulus, complex viscosity and tan delta are reported as the mean value \pm the standard deviation (SD).

The study was an open, single-center clinical trial conducted on 22 healthy female subjects under dermatological control. The protocol was approved by the Independent Derming Ethical Committee and registered on ClinicalTrial. gov (NCT03273660). The study was performed according to the guidelines of the Declaration of Helsinki relative to the Ethical Principles for Medical Research involving Human Subjects. All the participants gave their informed consent for participation as well as for the publication of the images. The mean age of the subjects was 50 years (range $42-60$ years). To be included in the study, volunteers had to meet the following inclusion criteria: female sex; age between 40 and 65 years; Face Volume Loss Scale (FVLS) grade between 2 and 4; no exposure of the face to strong UV radiation during the entire study duration; and the maintenance of habits in terms of food intake, physical activity and cosmetic use. At each planned visit, the volunteers had to present without make-up. Subjects were excluded if they were pregnant; were lactating; were smokers; were drug or alcohol abusers; were not in menopause but were without adequate contraceptive precautions or refused to perform pregnancy tests before esthetic procedures; showed a variation in BMI $( \pm 1)$ during the study period; underwent within the last 6 months nonpermanent esthetic corrections (including face lifts, laser treatments, Botox injections, chemical peels or biomaterial implants) or semipermanent fillers; or had in the past received permanent fillers. In addition, subjects were excluded from the study if they had diseases such as diabetes; endocrine disease; renal, cardiac or hepatic disorders; pulmonary disease; cancer; neurological disease; inflammatory or immunosuppressive disease; or allergies. Additional exclusion criteria were the use of anticoagulants and antiplatelet drugs, anti-histaminic drugs, corticosteroids (both topical and systemic), narcotics, antidepressants, immunosuppressive drugs (as part of hormonal or contraceptive treatments that started more than 1 year before the study) and, in general, drugs judged by the investigator as potentially interfering with the results.

The study was organized into 6 visits: one at baseline (T0), in which clinical and instrumental evaluations were performed, followed by the first injection procedure; one 3-4 weeks after the first injection procedure (T1), in which, again, clinical and instrumental evaluations were performed, followed by a touch-up treatment if needed, to treat possible asymmetries; a third visit (T2), in which clinical and instrumental evaluation was carried out 1 month after touch-up treatment; and then clinical and instrumental assessments were performed at 3 (T3), 6 (T4) and 9 (T5) months after the first injection procedure.

The injections $(0.4-1.1 \mathrm{~mL}$ of study product for each hemiface) were performed using needles or cannulas. Needles (25-27 G) were inserted perpendicular to the skin, and the volume was injected in three sites in contact with the periosteum on each side of the face, forming the shape of a pyramid to give greater structural anchoring and to minimize injections. When the cannula ( $25 \mathrm{G}, 40 \mathrm{~mm}$ ) was used, it was inserted in the deep tissue layers, and the product was deposited at the lateral zygomatic bone level 
in a fan-shaped pattern. The touch-up treatment (0.4-1.1 mL of study product) was performed to correct possible asymmetries 3-4 weeks after the first injection.

Qualitative (clinical) and quantitative (instrumental) assessments of the efficacy of the treatment were performed at each visit monolaterally (right or left side), according to the injection side randomization list made by the investigator before the inclusion of the subjects. The Wrinkle Severity Rating Scale (WSRS) ${ }^{15}$ was used to clinically assess nasolabial folds, while the Facial Volume Loss Scale (FVLS) ${ }^{16}$ was used for cheek ptosis. Five different grades are considered for both scales, as defined in Table 1.

For photographic documentation and midfacial volume instrumental evaluations, 3D pictures were taken with the VECTRA H1 handheld imaging system. The Vectra analysis module (VAM) was used to merge and compare the 3D pictures taken at two different times. Pictures were taken with standardized methods to guarantee comparable images, maintaining the same distance from the subject and the same illumination source intensity. All subjects were acclimatized under relaxing conditions for at least 10-15 minutes before each visit.

A subjective evaluation of the treatment was recorded at T5 with the completion of a questionnaire in which efficacy and unpleasant effects were considered.

The presence of eventual undesired or adverse events was checked by the investigator.

Statistical analysis was performed with a nonparametric test (Wilcoxon test) for clinical assessment. For instrumental data, nonparametric tests were used when the normality hypothesis was rejected by the Shapiro-Wilk test at a threshold lower than $5 \%$. When the hypothesis was confirmed, parametric tests were used.

\section{Results}

The study product exhibited a G' value equal to $295 \pm 23 \mathrm{~Pa}$ and a tan delta of $0.12 \pm 0.02$ (at $1.59 \mathrm{~Hz}$ frequency, within the LVR) (Table 2). The value for complex viscosity was $69 \pm 2 \mathrm{~Pa} \times \mathrm{s}(0.7$ $\mathrm{Hz}$ frequency). The rheological parameters fall within the values registered for "volumetric" gels with a high projection capacity, which is consistent with the ASV clinical indications and suggested plan of injection. ${ }^{17}$

During the study, 6 patients dropped out of the study for reasons not related to the study. Data analysis was, therefore, performed on a total of 20 patients up to T2, on 18 patients at $\mathrm{T} 3$ and 16 patients at T4 and T5. The mean volume of filler injection was $0.68 \mathrm{~mL}$ at $\mathrm{T} 0$ and $0.42 \mathrm{~mL}$ at $\mathrm{T} 1$, while the number of touch-up injections was 20 at T1. Starting already
Table I WSRS and FVLS Grades Used for Clinical Evaluation of the Efficacy

\begin{tabular}{|c|c|c|}
\hline Grade & WSRS & FVLS \\
\hline I & $\begin{array}{l}\text { No visible nasolabial folds; } \\
\text { continuous skin line }\end{array}$ & $\begin{array}{l}\text { Mild flattening or } \\
\text { shadowing of one or more } \\
\text { facial regions (including the } \\
\text { cheek, temple, preauricular } \\
\text { and periorbital areas). No } \\
\text { prominent bony landmarks. } \\
\text { No visibility of underlying } \\
\text { musculature }\end{array}$ \\
\hline 2 & $\begin{array}{l}\text { Shallow but visible } \\
\text { nasolabial folds with a slight } \\
\text { indentation; minor facial } \\
\text { feature }\end{array}$ & $\begin{array}{l}\text { Intermediate point between } \\
\text { grade I and grade } 3\end{array}$ \\
\hline 3 & $\begin{array}{l}\text { Moderately deep nasolabial } \\
\text { folds; clear facial feature } \\
\text { visible at normal } \\
\text { appearance but not when } \\
\text { stretched }\end{array}$ & $\begin{array}{l}\text { Moderate concavity of one } \\
\text { or more facial regions } \\
\text { (including the cheek, } \\
\text { temple, preauricular and } \\
\text { periorbital areas). } \\
\text { Prominent bony landmarks. } \\
\text { May have visibility of } \\
\text { underlying musculature }\end{array}$ \\
\hline 4 & $\begin{array}{l}\text { Very long and deep } \\
\text { nasolabial folds; prominent } \\
\text { facial feature; <2 } \mathrm{mm} \text { folds } \\
\text { visible when stretched }\end{array}$ & $\begin{array}{l}\text { Intermediate point between } \\
\text { grade } 3 \text { and grade } 5\end{array}$ \\
\hline 5 & $\begin{array}{l}\text { Extremely long and deep } \\
\text { nasolabial folds; detrimental } \\
\text { to facial appearance; } \\
2-4 \mathrm{~mm} \text { V-shaped folds } \\
\text { visible when stretched }\end{array}$ & $\begin{array}{l}\text { Severe indentation of one } \\
\text { or more facial regions } \\
\text { (including the cheek, } \\
\text { temple, preauricular and } \\
\text { periorbital areas). Severe } \\
\text { prominence of bony } \\
\text { landmarks. Clear visibility of } \\
\text { underlying musculature }\end{array}$ \\
\hline
\end{tabular}

Table 2 Rheological Parameters for the Gel

\begin{tabular}{|l|l|l|}
\hline G' $(\mathbf{P a})$ & Complex Viscosity $(\mathbf{P a} \times \mathbf{s})$ & Tan Delta (G'/G') \\
\hline $295 \pm 23$ & $69 \pm 2$ & $0.12 \pm 0.02$ \\
\hline
\end{tabular}

Notes: The storage modulus $\left(G^{\prime}\right)$ and the tan delta values within the linear viscoelastic range, and the complex viscosity value at $0.7 \mathrm{~Hz}$ frequency are reported. Measurements were carried out at $37^{\circ} \mathrm{C}$.

from T1 (ie, after the first injection procedure), and lasting up to T5, a significant improvement in cheek volume was found. In detail, the FVLS mean value was reduced by $22.2 \%$ at $\mathrm{T} 1$, $25.9 \%$ at $\mathrm{T} 2,37 \%$ at $\mathrm{T} 3,33.3 \%$ at T 4 and $22.2 \%$ at T5 (Figure 1). The values were all statistically significant (Wilcoxon test, $\mathrm{p}<0.05, \mathrm{p}<0.01$ or $<0.001)$ compared to T0. Importantly, 
a decrease in the clinical score of at least 1 grade was reported in $55 \%, 65 \%, 83 \%, 75 \%$ and $44 \%$ of the evaluated subjects at T1, T2, T3, T4 and T5, respectively. A statistically significant reduction of wrinkle severity was reported at $\mathrm{T} 1$ (11.5\%) and T2 (15.4\%). The clinical reduction was maintained at the subsequent visits of T3 (15.4\%), T4 (15.4\%) and T5 (8.7\%), although in this case, the reduction did not reach a statistically significant $\mathrm{p}$ value (Figure 2 ). This corresponded to a reduction in the clinical score of at least 1 grade in 30\%, 45\%, 50\%, 44\% and $25 \%$ of the subjects (at T1, T2, T3, T4 and T5, respectively).

To determine the increased facial volume after injections, 3D pictures taken at each visit were analyzed using the VAM, which is able to merge and compare images taken at different times and to calculate the differences in volume. The results of these analyses (reported in Figure 3) showed an average increase in volume already well determined at T1 (0.916 cc versus T0) that further increased at T2 (1.275 cc) and T3 (1523 cc) and was maintained at T4 $(1.153 \mathrm{cc})$ and even at T5 $(1.107 \mathrm{cc})$. From these data, it was possible to calculate that $35 \%$ of subjects had a very important improvement in cheek facial volume greater than $1 \mathrm{cc}$ at $\mathrm{T} 1$, and this percentage increased to $75 \%$ after $\mathrm{T} 2$ (touch-up) and was maintained at all the other time points of the study $(67 \%, 62 \%$ and $50 \%$ at T3, T4 and T5, respectively).

An important observation is that if the data are compared for each time point of the study in terms of variation percentage ( $\Delta \mathrm{Tx}-\mathrm{T} 0$ vs $\Delta \mathrm{T} 1-\mathrm{T} 0)$, it is evident that there not only is an important volumizing activity but also that this positive activity is persistent throughout the entire study period (Figure 3 ).

Figure 4 reports a representative sequence of images from a single subject (subject 4) taken at different time points of the study, and the subsequent VAM analysis of the same images from the same subject, using a color distance map, is shown in Figure 5. From both figures, it is possible to appreciate the improvement following the injection of the study product.

The injections were well tolerated by the subjects, some of whom reported the appearance of light bruises or swelling at the injection point or small nodules ascribable to the accumulation of the product. All these slight events disappeared in 10-15 days and were considered to be due to a nonspecific skin reaction; the final scoring from both the investigator and the subject was good and excellent tolerability for all the subjects. None of the reported undesired effects were considered an adverse event by investigators. The self-assessment questionnaire evaluations, analyzed as

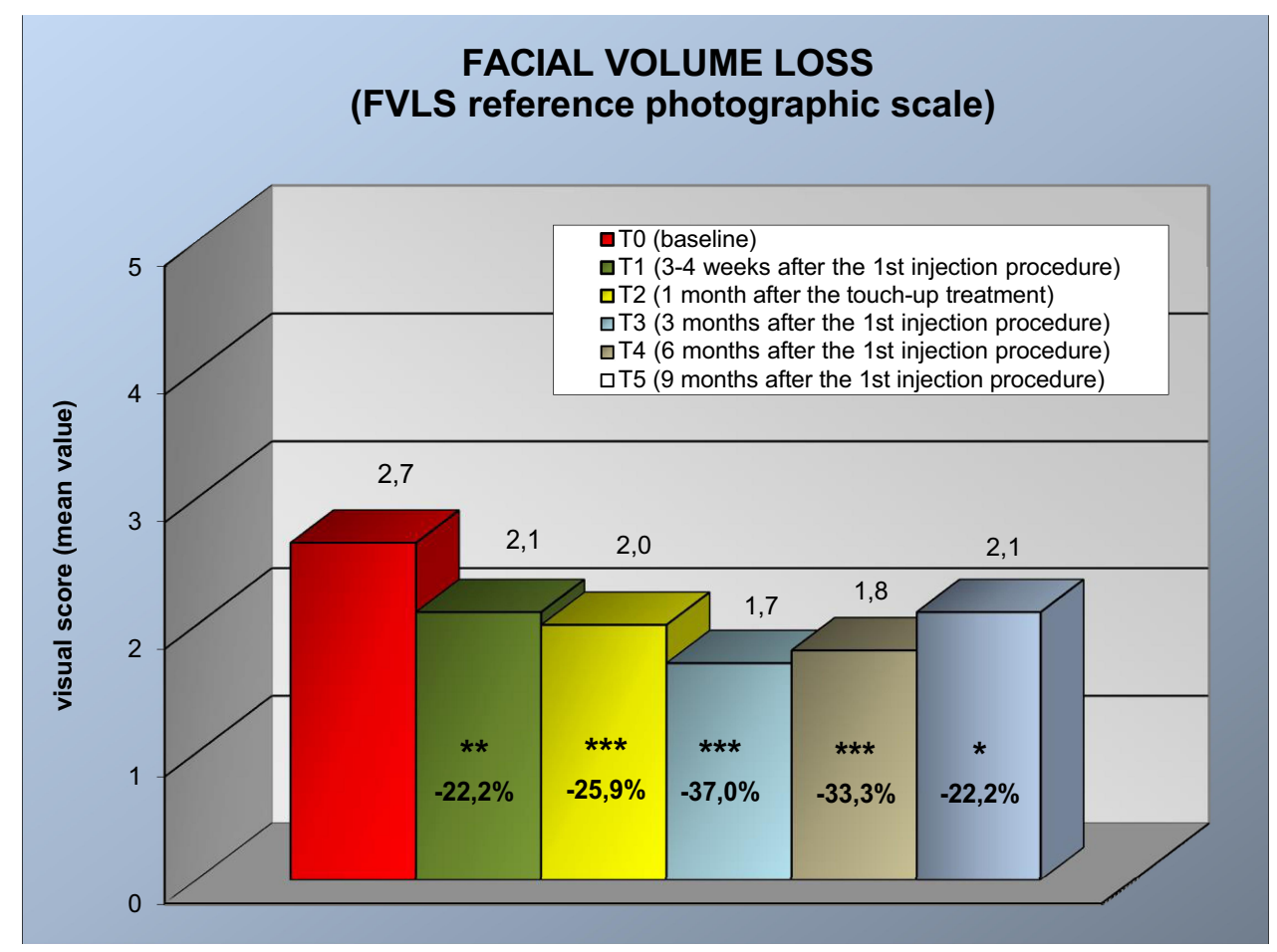

Figure I Clinical evaluation of facial volume loss (FVLS reference photographic scale) at the different time points. ${ }^{*} p<0.05$, $* *<<0.0$ I, $* * * p<0.00 I$ vs T0 (Wilcoxon test). 


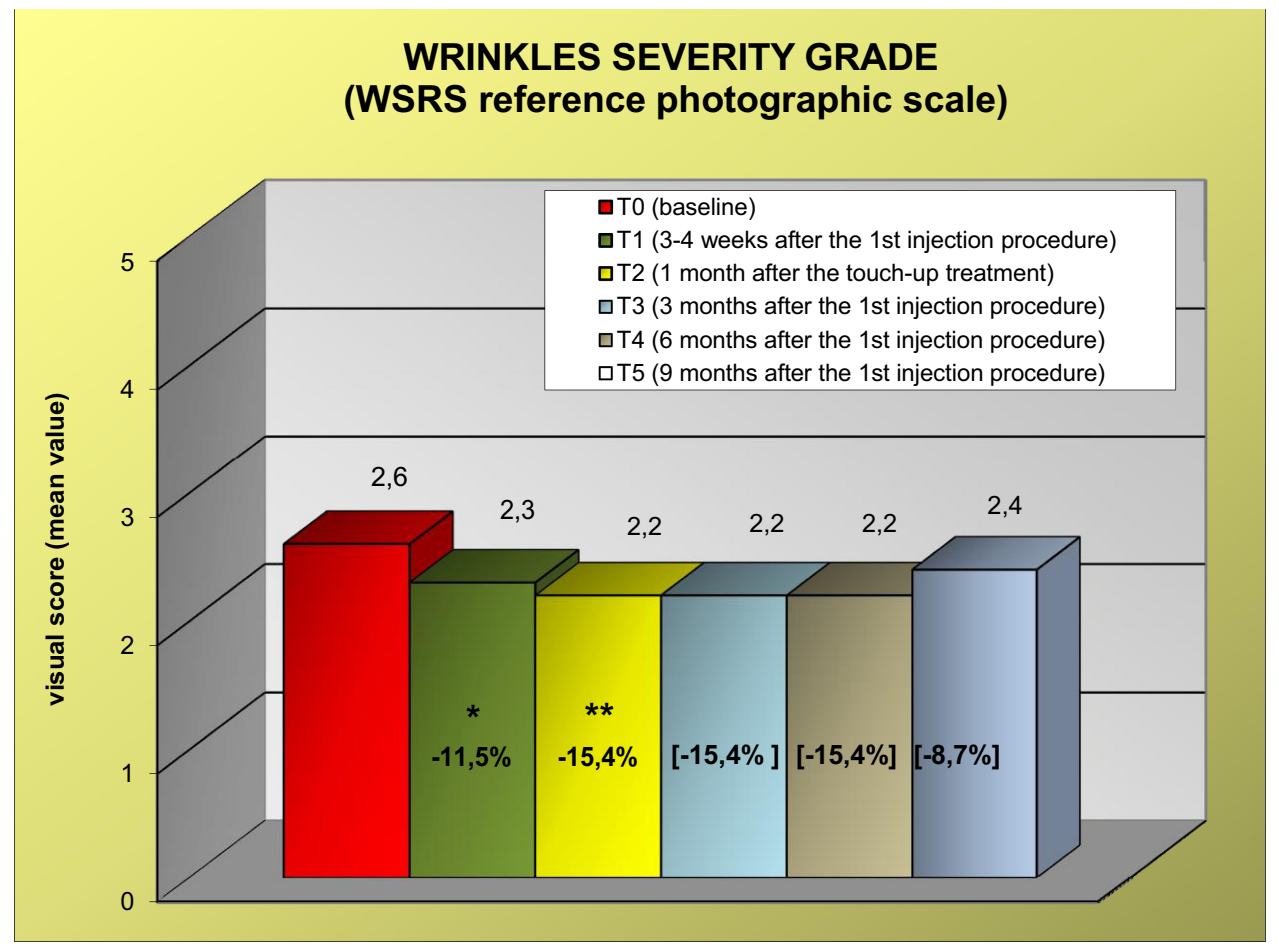

Figure 2 Clinical evaluation of wrinkle severity grade (WSRS reference photographic scale) at the different time points. The values of the score are shown above the boxes, while the values in percentages inside each box represent the percentage of the decrease in severity. ${ }^{*} p<0.05$, ${ }^{* *} p<0.01$, vs T0 (Wilcoxon test).

the sum of medium, marked and very marked judgments, are summarized in Table 3. It is clear that the subject judgment was more than positive, particularly considering the cheek volume and skin suppleness.

\section{Discussion}

For esthetic facial treatments, particularly in those that are age-related, HA is undoubtedly the most widely used agent. It has shown clinical efficacy in the treatment of

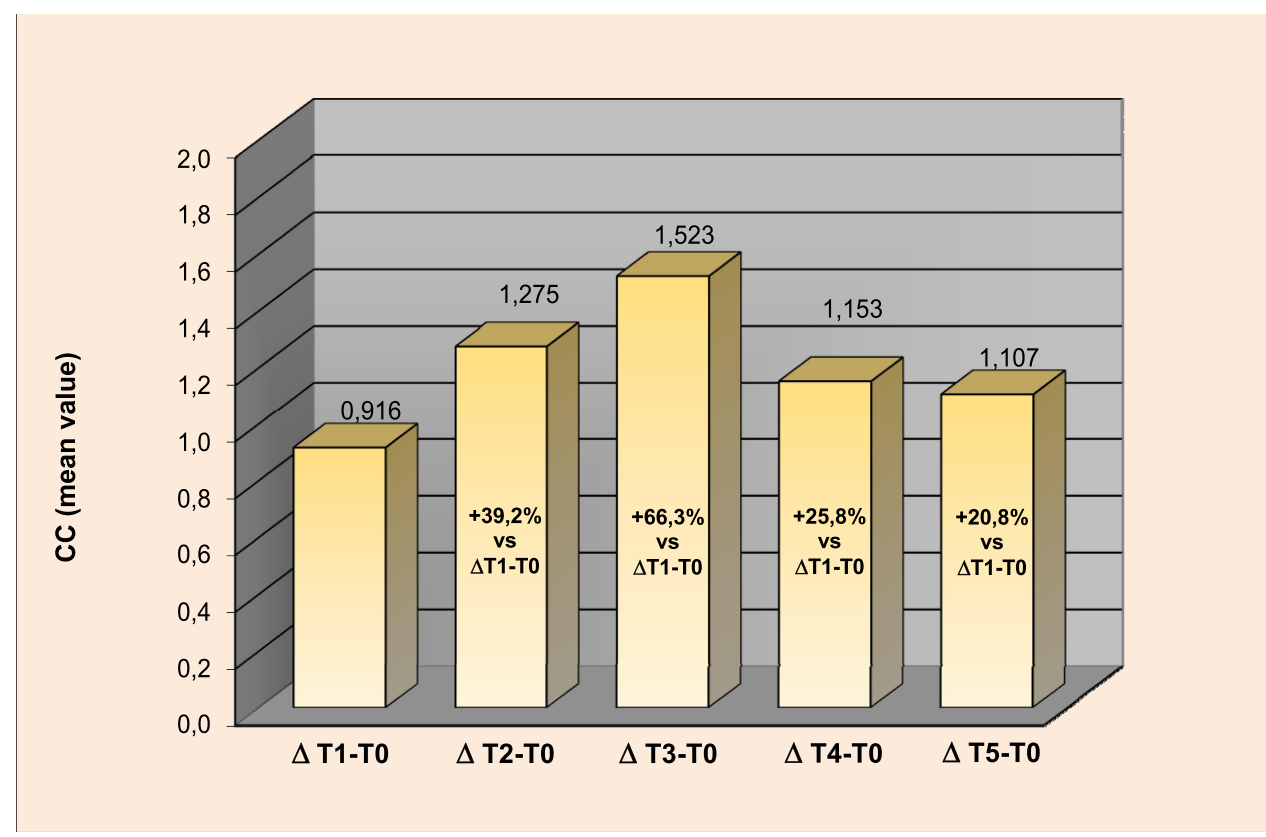

Figure 3 Instrumental evaluation of cheek volumes by 3D facial volume imaging analysis. The figure shows the change in volume (in cc) at the different time points. The exact figures are reported above the boxes, while the percentages inside each box report the $\Delta$ variation for each time point compared to the $\Delta$ variation at TI-T0. 


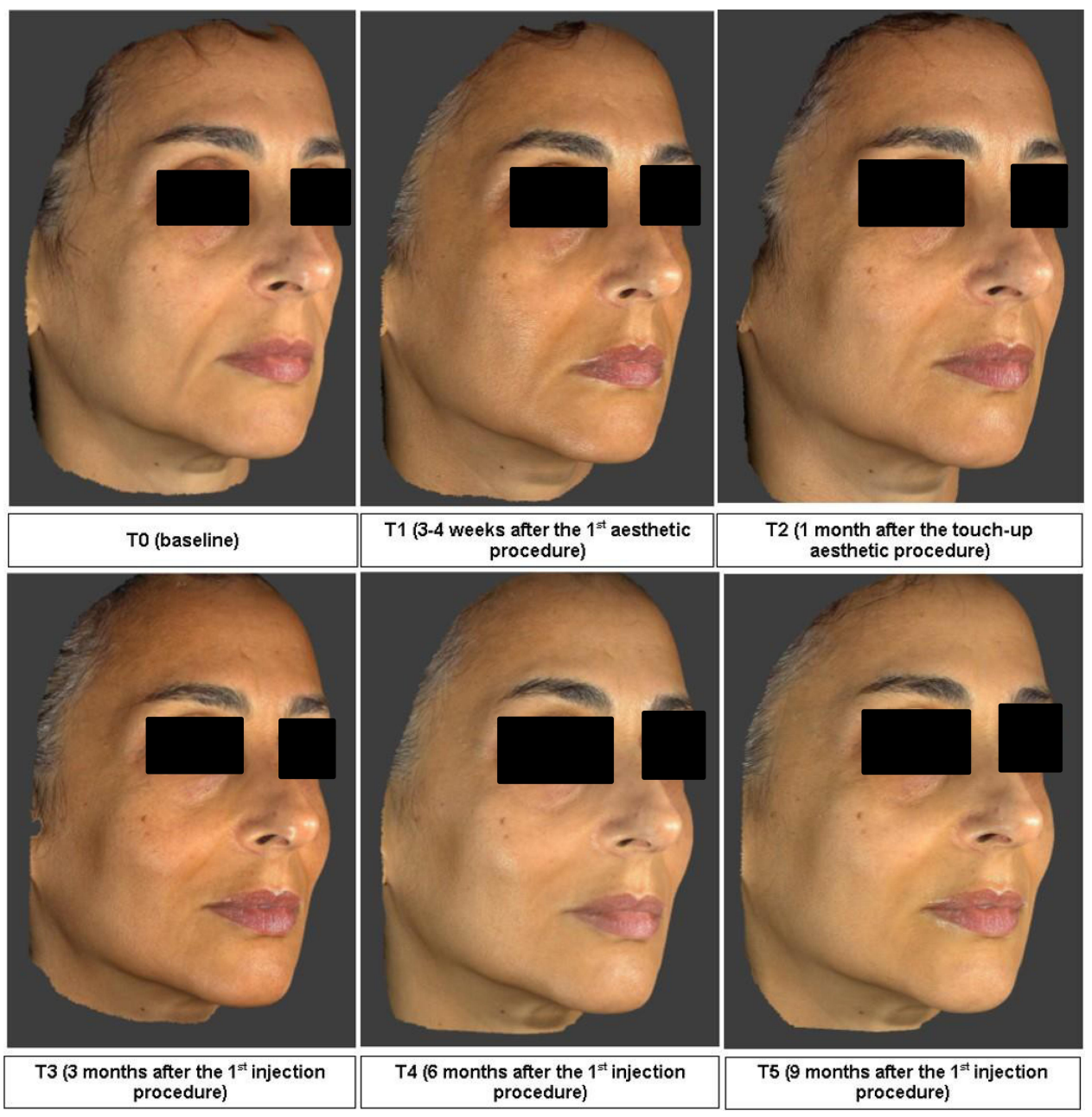

Figure 4 Representative 3D images of subject 4 obtained with Vectra $\mathrm{HI}$ at T0 and at all the other time points.

several deep or superficial facial defects and represents, for several reasons, an ideal dermal filler. ${ }^{9,11-13,18}$ In fact, it has good tolerability and is biocompatible. It is available in several preparations with different HA contents and natures with the aim of fulfilling the specific requests for the different treatments. Crosslinked HA has shown activity as a volumizer filler, a property that renders it the ideal compound for age-related midfacial volume defect treatment. Indeed, volume loss is one of the main reasons for skin sagging and the deepening of nasolabial folds. ${ }^{4,9,10,13}$ The clinical effect of a dermal filler and revolumizing medical device should not only be clear and measurable but also long-lasting and associated with good tolerability.

The product under study has been characterized in terms of rheological behavior. Parameters such as the storage modulus (G'), the tan delta (G'/G') and the complex viscosity are key features defining a dermal filler. $G^{\prime}$ is a measure of gel rigidity/deformability and is related to filler projection capacity: higher $G^{\prime}$ values are related to lower deformability and higher projection ability. Tan delta is a measure of the extent of gel elasticity and of its tendency to behave as an elastic solid under a certain stress: the lower the tan delta values, the higher the elasticity. Gel viscosity is a measure of gel resistance to flow, thus affecting its ability to spread into the tissue beyond the ease of delivery. Overall, the rheological characteristics of a filler are crucial for its performance in terms of volumizing effect and, along with cohesivity, of tissue integration. The tested gel presents a G' compatible with similar commercialized volumetric fillers but, differently from those, present better cohesivity. ${ }^{19}$ This is important, as generally, this latter parameter correlates well with the tissue integration pattern. ${ }^{19}$

The present study was performed in female volunteers with age-related midfacial volume defects, and the results clearly show that the product is effective in ameliorating facial volume defects and that this effect is observable after 


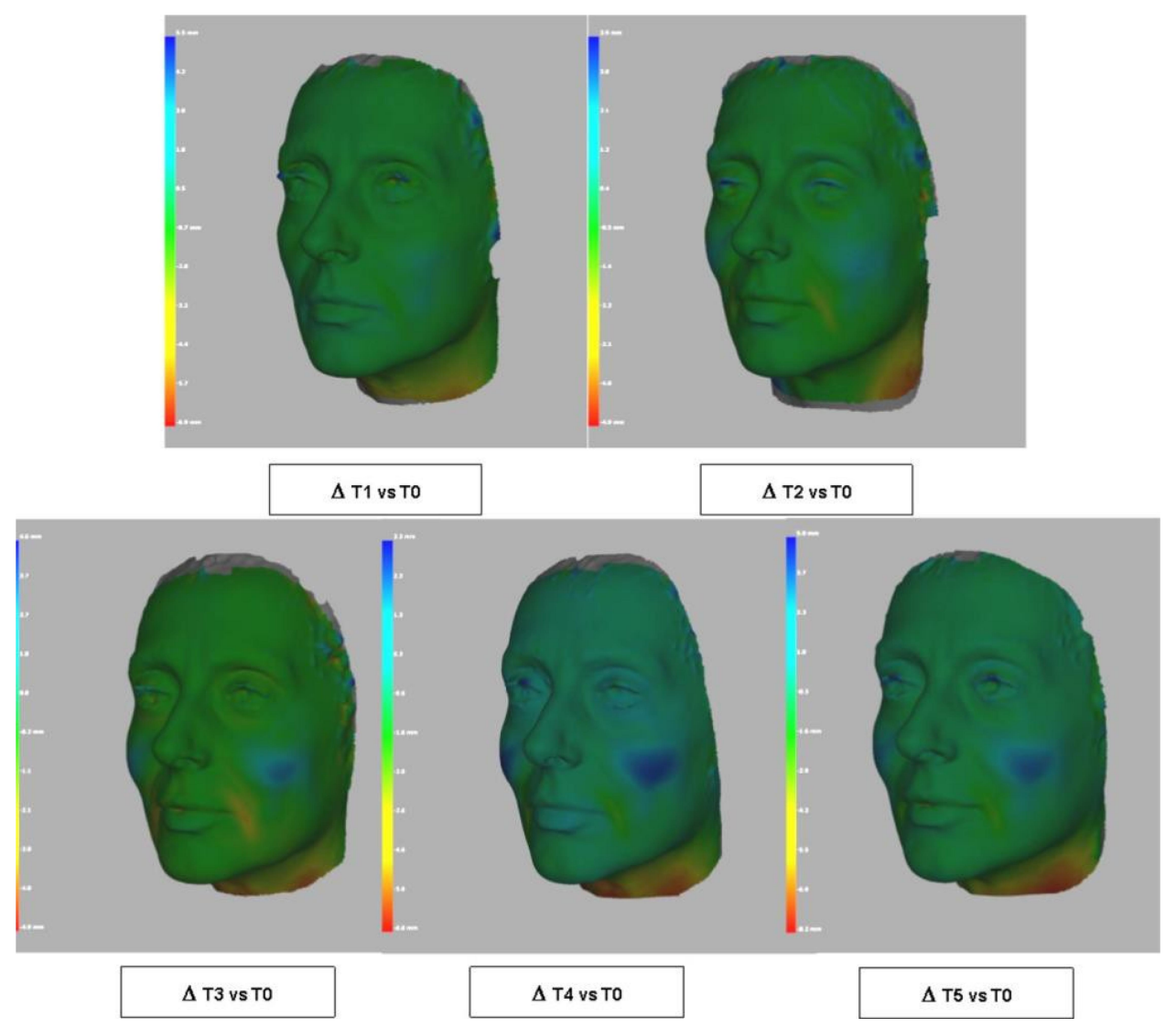

Figure 5 VAM analysis (color distance map) of facial volume obtained from images reported in Figure 4.

the first injection and maintained for at least 9 months, the endpoint of the present study. The preparation has, in fact, shown, at all the prefixed time points of the study, both a significant improvement in FVLS score (revolumizing effect) and a significant reduction in WSRS score (antiwrinkling effect). The study was planned to end with the fifth visit at 9 months from the first injection, a time frame sufficiently long to demonstrate the long-lasting effect of the

Table 3 Self-Assessment by Subjects Conducted with the Completion of a Questionnaire at T5

\begin{tabular}{|l|l|}
\hline & $\begin{array}{l}\text { Sum of Medium, Marked and } \\
\text { Very Marked Judgments (\%) }\end{array}$ \\
\hline Improvement in cheek volume & 69 \\
Reshaping of face silhouette & 57 \\
Reduction of deep wrinkles & 44 \\
Reduction of superficial wrinkles & 63 \\
Lifting effect & 50 \\
Improvement of skin suppleness & 69 \\
Improvement of skin smoothness & 63 \\
Improvement of skin brightness & 56 \\
Improvement of skin hydration & 50 \\
\hline
\end{tabular}

study product. We cannot exclude that this activity could be maintained for even longer times, although this would require additional studies to be formally proven.

The efficacy and long-lasting action of the study product was not only clinically demonstrated by the investigator but also fully supported by instrumental (3D images and VAM analysis) data, which further corroborate the results. Moreover, the questionnaires completed by the majority of the volunteers at the end of the study underlined the highvolumizing performance of the treatment, further strengthening the results obtained. Notably, positive efficacy results have been obtained with very good tolerability of the product.

A possible limitation of the study is the relatively small number of subjects, although the variability in the results among them was very limited. A second point that we did not consider was the seasonality of the results, as the study was performed only in the period between June and September. However, we can speculate that this is probably the period in Italy, if any, in which we could expect a lower efficacy of the application. Perhaps a study extension to other periods of the year would have even enhanced the positivity of the results. 


\section{Conclusion}

In conclusion, the study performed in women with agerelated midfacial volume defects treated with the study product showed a clear and long-lasting esthetic performance of the product, which was maintained for 9 months of evaluation. The treatment was able to ameliorate the depth of folds, volume loss and wrinkles with very good tolerability.

\section{Data Sharing Statement}

The de-identified data of the Clinical Study Report (CSR) are made available upon request to corresponding author for five years.

\section{Acknowledgments}

The authors are grateful to Giovanna Damia for help in writing the manuscript. This study has been sponsored by IBSA Farmaceutici Italia.

\section{Disclosure}

AMG, GV and GB are employees of IBSA Farmaceutici Italia. AS, BT and LLP are employees of DERMING S.r.l. Professor Chiara Schiraldi reports grants from Bioteknet, outside the submitted work. The authors report no other conflicts of interest in this work.

\section{References}

1. Furnas DW. The retaining ligaments of the cheek. Plast Reconstr Surg. 1989;83(1):11-16. doi:10.1097/00006534-198901000-00003

2. Jacono AA, Ranson ER. Anatomic predictors of unsatisfactory outcomes in surgical rejuvenation of the midface. JAMA Facial Plast Surg. 2013;15(2):101-109. doi:10.1001/jamafacial.2013.443

3. Warren R, Gartstein V, Kligman AM, Montagna W, Allendorf RA, Ridder GM. Age, sunlight, and facial skin: a histologic and quantitative study. $J$ Am Acad Dermatol. 1991;25(5,Part 1):751-760. doi:10.1016/S0190-9622(08)80964-4

4. Donath AS, Glasgold RA, Glasgold MJ. Volume loss versus gravity: new concepts in facial aging. Curr Opin Otolaryngol Head Neck Surg. 2007;15(4):238-243. doi:10.1097/MOO.0b013e32825b0751

5. Downs BW, Wang TD. Current concepts in midfacial rejuvenation. Curr Opin Otolaryngol Head Neck Surg. 2008;16(4):335-338. doi:10.1097/MOO.0b013e3283079c9b
6. Rohrich RJ, Pessa JE. The fat compartments of the face: anatomy and clinical implications for cosmetic surgery. Plast Reconstr Surg. 2007;119(7):2219-2227. doi:10.1097/01.prs.0000265403.66886.54

7. Farkas JP, Pessa JE, Hubbard B, Rohrich RJ. The science and theory behind facial aging. Plast Reconstr Surg Glob Open. 2013;1(1):e8e15. doi:10.1097/GOX.0b013e31828ed1da

8. Glogau RG. Aesthetic and anatomic analysis of the aging skin. Semin Cutan Med Surg. 1996;5(3):134-138. doi:10.1016/S1085-5629(96) 80003-4

9. Callan P, Goodman GJ, Carlisle I, et al. Efficacy and safety of a hyaluronic acid filler in subjects treated for correction of midface volume deficiency: a 24 month study. Clin Cosmet Investig Dermatol. 2013;6:81-89. doi:10.2147/CCID.S40581

10. Humphrey S, Beleznay K, Fitzgerald R. Combination therapy in midfacial rejuvenation. Dermatol Surg. 2016;42:S83-S88. doi:10.1097/DSS.0000000000000747

11. Benjamin A, Christiane B, Patrick B, et al. Efficacy and safety of a new hyaluronic acid dermal filler in the treatment of severe nasolabial lines - 6-month interim results of a randomized, evaluatorblinded, intra-individual comparison study. J Cosmet Dermatol. 2011;10(2):94-98. doi:10.1111/j.1473-2165.2011.00550.x

12. Hong JY, Choi EJ, Choi SY, Li K, Kim BJ. Randomized, patient/ evaluator-blinded, intraindividual comparison study to evaluate the efficacy and safety of a novel hyaluronic acid dermal filler in the treatment of nasolabial folds. Dermatol Surg. 2018;44(4):542-548. doi:10.1097/DSS.0000000000001335

13. Jones D, Murphy DK. Volumizing hyaluronic acid filler for midface volume deficit: 2-year results from a pivotal single-blind randomized controlled study. Dermatol Surg. 2013;39(11):1602-1612. doi:10. $1111 /$ dsu. 12343

14. Tezel A, Fredrickson GH. The science of hyaluronic acid dermal fillers. J Cosmet Laser Ther. 2008;10(1):35-42. doi:10.1080/14764 170701774901

15. Day DJ, Littler CM, Swift RW, Gottlieb S. The wrinkle severity rating scale. Am J Clin Dermatol. 2004;5(1):49-52. doi:10.2165/ 00128071-200405010-00007

16. Lorenc ZP, Bank D, Kane M, Lin X, Smith S. Validation of a four-point photographic scale for the assessment of midface volume loss and/or contour deficiency. Plast Reconstr Surg. 2012;130 (6):1330-1336. doi:10.1097/PRS.0b013e31826d9fa6

17. La Gatta A, Papa A, Schiraldi C, De Rosa M. Hyaluronan dermal fillers via crosslinking with 1,4-butandiol diglycidyl ether: exploitation of heterogeneous reaction conditions. J Biomed Mater Res B Appl Biomater. 2016;104(1):9-18. doi:10.1002/jbm.b.33329

18. Baumann L, Weiss RA, Grekin S, et al. Comparison of hyaluronic acid gel with (HARDL) and without lidocaine (HAJUP) in the treatment of moderate-to-severe nasolabial folds: a randomized, evaluator-blinded study. Dermatol Surg. 2018;44(6):833-840. doi:10.1097/DSS.0000000000001424

19. La Gatta A, Salzillo R, Catalano C, et al. Hyaluronan-based hydrogels as dermal fillers: the biophysical properties that translate into a "volumetric" effect. PLoS One. 2019;14(6):e0218287-e0218287. doi:10.1371/journal.pone. 0218287
Clinical, Cosmetic and Investigational Dermatology is an international, peer-reviewed, open access, online journal that focuses on the latest clinical and experimental research in all aspects of skin disease and cosmetic interventions. This journal is indexed on CAS.
The manuscript management system is completely online and includes a very quick and fair peer-review system, which is all easy to use. Visit http://www.dovepress.com/testimonials.php to read real quotes from published authors. 